\title{
High spatial resolution capabilities of Doppler measurements with the Pic du Midi MSDP spectrograph
}

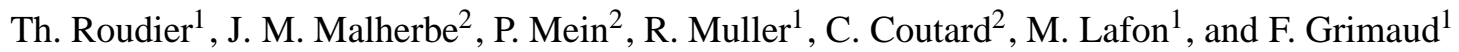 \\ ${ }^{1}$ Laboratoire d'Astrophysique de Toulouse, Observatoire Midi-Pyrénées, 14 avenue É. Belin, 31400 Toulouse, France \\ ${ }^{2}$ LESIA, Observatoire de Paris, Section de Meudon, 92195 Meudon, France
}

Received 7 April 2003 / Accepted 3 July 2003

\begin{abstract}
We analyse observations in the NaD1 line ( $\lambda 5896 \AA$ ) obtained with the MSDP spectrograph of the Turret Dome of the Pic du Midi Observatory. Individual images reveal high spatial resolution and the data reduction shows the high capabilities of the spectrograph to get doppler measurements up to the limit of the resolution of the refractor $(0 ; 3)$. Dopplershifts are obtained in the middle and high photosphere. The smallest granules 0.4 show upward motions in the middle photosphere.
\end{abstract}

Key words. Sun: photosphere - Sun: granulation

\section{Introduction}

The time analysis of the plasma and magnetic field on the Sun surface requires high spatial resolution observations $\left(0,{ }^{\prime \prime} 2\right.$ or better). The combination of the high spatial and spectral resolutions proves to be a challenge for observational solar physics (Bonet 1999). This challenge arises because of the basic properties of the complex interactions between hydrodynamics, radiative transfer, magnetic field at the sun surface. The MSDP spectrograph is a good compromise to determine the profile of one or more lines at every point in the two-dimensional field of view. From these profiles and relative wavelength dependent contributions of the different atmospheric layers, one can extract fluctuations of intensity and velocity measured at different depths in the solar atmosphere. The NaD1 line is particularly interesting for photospheric diagnostic since its extended wings allow us to obtain information from a wide range of heights through the photosphere. The new CCD camera which has been installed at the MSDP spectrograph of the Turret Dome of the Pic du Midi Observatory helps us to reduce the exposure time down to $20 \mathrm{~ms}$ allowing to freeze the atmospheric seeing and get high spatial observation close to $0 . ' 3$ of resolution (1 pixel $=00^{\prime \prime} 13$, Fig. 1).

We present in this paper the capabilities of the MSDP spectrograph installed at the Pic du Midi which allows to get dopplergrams approaching the limit of the resolution of the refractor $(0, ' 3$ defined as the width at half maximun of the Airy function at $\lambda 5896 \AA$ ) over a large field of view.

Send offprint requests to: Th. Roudier, e-mail: roudier@bigorre. bagn.obs-mip.fr

\section{Observations and data processing}

The observations were performed in the NaD1 line $(\lambda 5896 \AA)$ with the Multichannel Subtractive Double Pass (MSDP) system designed by P. Mein, operating on the solar spectrograph as designed by Z. Mouradian. The MSDP is installed at the focus of the $50 \mathrm{~cm}$ refractor of the Pic du Midi Observatory (Coupole Tourelle) as described by Mein $(1981,1999,2002)$. The observation was done at disc center on 1st october 2002 (between $9 \mathrm{~h} 01 \mathrm{~m} 13 \mathrm{~s}$ and $9 \mathrm{~h} 42 \mathrm{~m} 54 \mathrm{~s}$ UT). The exposure time was $20 \mathrm{~ms}$ with Camera LaVision CCD $(1280 \times 1024$ pixels $)$. The pixel size on the CCD camera was 0 .' 13 . The spectral resolution is $144 \mathrm{~m} \AA$ and 11 channels were recorded simultaneously. The elementary field of view (FOV) is $90^{\prime \prime} \times 14^{\prime \prime}$ (Fig. 1), but a larger FOV can be obtained sequentially by moving the image of the sun in front of the entrance window of the spectrograph up to $90^{\prime \prime} \times 90^{\prime \prime}$.

In the present study, velocity $(V)$ and intensity $(I)$ maps have been computed in the wings of the profile where the halfwidth is $\delta \lambda=288 \mathrm{~m} \AA$. The location of the atmospheric height corresponding to this chord has been estimated by Eibe (2001) and Roudier (2001) to be $170 \mathrm{~km}$ for velocity $(V)$ and $130 \mathrm{~km}$ for the intensity $(I)$ which represents middle photosphere. In addition to $V$ and $I$ in the wing of the line, we consider approximate values of the "continuum intensity" deduced directly from one extreme channel of the raw data where the spatial resolution is maximum, because such maps do not suffer any wavelength interpolation between several channels.

The standard corrections for atmospheric transparence variations, dark-current, flat-field, and correction of the errors of interpolations have been computed. The intensity and Doppler velocity were computed by using the bisector technique (lambdameter technique) at $\delta \lambda=288 \mathrm{~m} \AA$. 


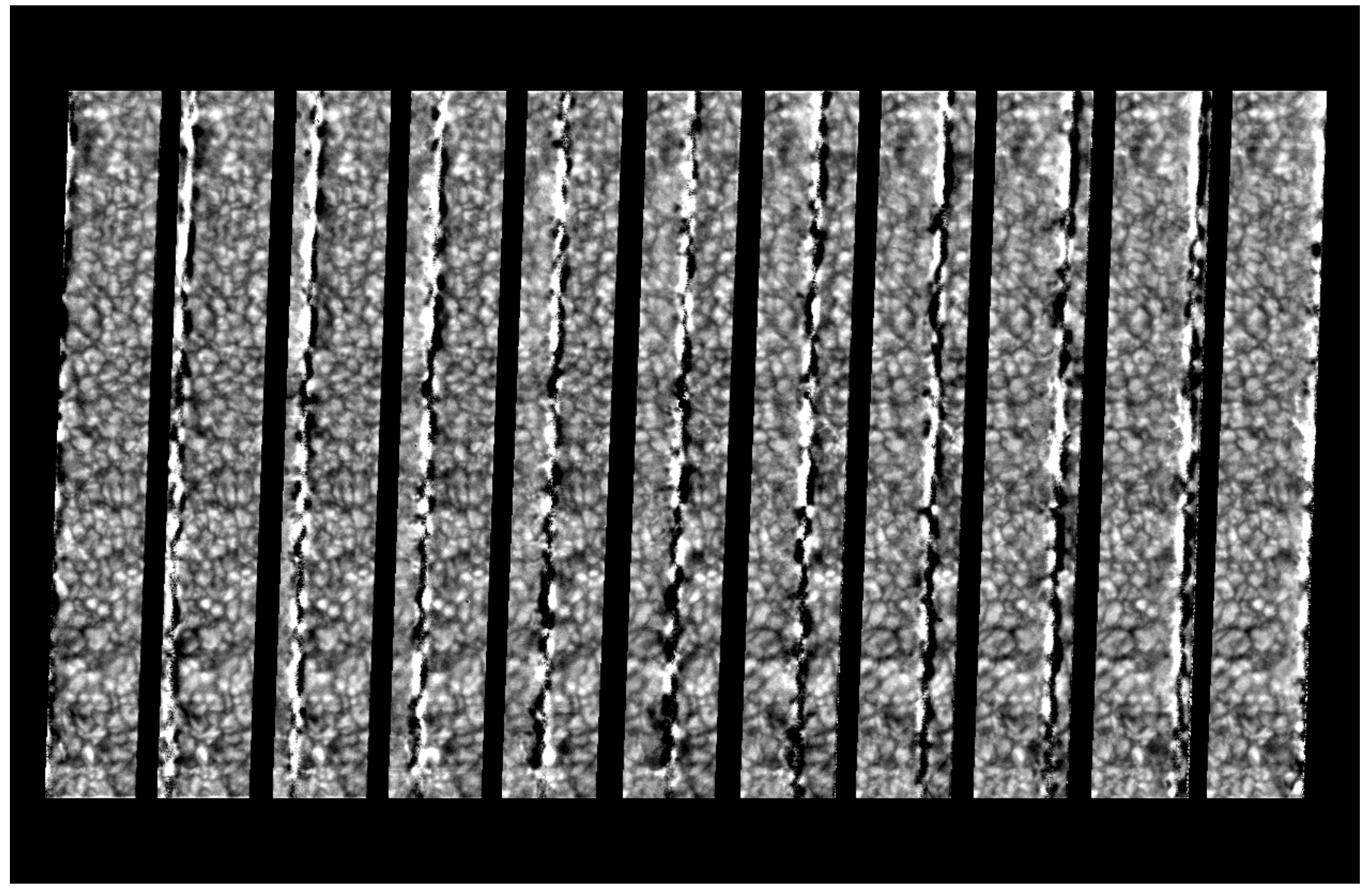

Fig. 1. Example of MSDP spectrum NaD1 $5896 \AA$ at $09 \mathrm{~h} 36 \mathrm{~m} 15 \mathrm{~s}$ UT. The field of view is $90^{\prime \prime} \times 14^{\prime \prime}$. The spectral shift between each channel is $144 \mathrm{~m} \AA$. The wavelength varies linearly from the left to the right of each channel. Numerous very small features close to the limit of the resolution of the refractor $(0,3)$ are visible in the different channels of this raw spectrum.

In the following velocity and intensity maps have been plotted for the observation taken at $9 \mathrm{~h} 36 \mathrm{~m} 15 \mathrm{~s}$ and $9 \mathrm{~h} 36 \mathrm{~m} 18 \mathrm{~s}$. The field of view is $6^{\prime \prime} \times 76^{\prime \prime}$.

\section{Velocity an intensity maps: resolution of the observation}

We present in Figs. 2 and 3 the most representative examples (at $9 \mathrm{~h} 36 \mathrm{~m} 15 \mathrm{~s}$ and $9 \mathrm{~h} 36 \mathrm{~m} 18 \mathrm{~s} \mathrm{UT}$ ) of continuum intensities, velocities and intensities measured at $\delta \lambda=288 \mathrm{~m} \AA$, middle photosphere, over a field of view of $6^{\prime \prime} \times 76^{\prime \prime}$. In the intensity image at $\delta \lambda=288 \mathrm{~m} \AA$ (top), we observe small bright points which subsist on several consecutive maps. These bright points are associated with the well known filigree. The location of some of these bright points (A and B) are indicated in Fig. 2 by arrows. The reached spatial resolution of our intensity and velocity maps is comparable to the ones obtained by speckle restoration at the VTT in Tenerife $\left(0{ }^{\prime \prime} 4\right)$ by Krieg et al. (2000), Hirzberger et al. (2001) and Hirzberger (2002).

In the velocity map (bottom of Fig. 2), the upflows are coded in white corresponding to positive Dopplershift. An arrow shows, in this figure, the smallest granule $\mathrm{C}$ which has a size of 0.4 (width at half maximun of the profile) very close to the diffraction limit of the refractor. The difference of spatial resolution between the measured resolution in the raw spectra shown in Fig. $1\left(0{ }^{\prime \prime} 3\right)$ and the limit of spatial resolution in Dopplershifts $\left(0\right.$.' $\left.^{\prime}\right)$ can be explained by interpolations done at the different steps of the data reduction.

In Fig. 2, the high correlation between the velocity map (bottom) and intensity continuum map (middle) is clearly visible. The difference in the appearence of the granules between the velocity (botttom) and intensity maps at $\delta \lambda=288 \mathrm{~m} \AA$ (top) is essentially due to the altitude at which these quantities are measured, where at this level, the intensity of granulation is vanishing.

Figures 4 and 5 show the intensity cross sections at $\delta \lambda=$ $288 \mathrm{~m} \AA$ and continuum, in the central part of the small feature A (i.e. Fig. 2) which demonstrates the high resolution of the observation.

Figure 6 exhibits the line profile of that small bright point $\mathrm{A}$ compared to the mean profile derived from all the profiles of the quiet sun. The line core of the feature A is more or less centered relative to the mean profile, but we must note that here the 5 min oscillations have not been removed.

\section{Vertical flow characteristics and granule dimension}

To characterize the flow, granule size as well as mean intensity and mean velocity, were derived. First, in order to reduce 

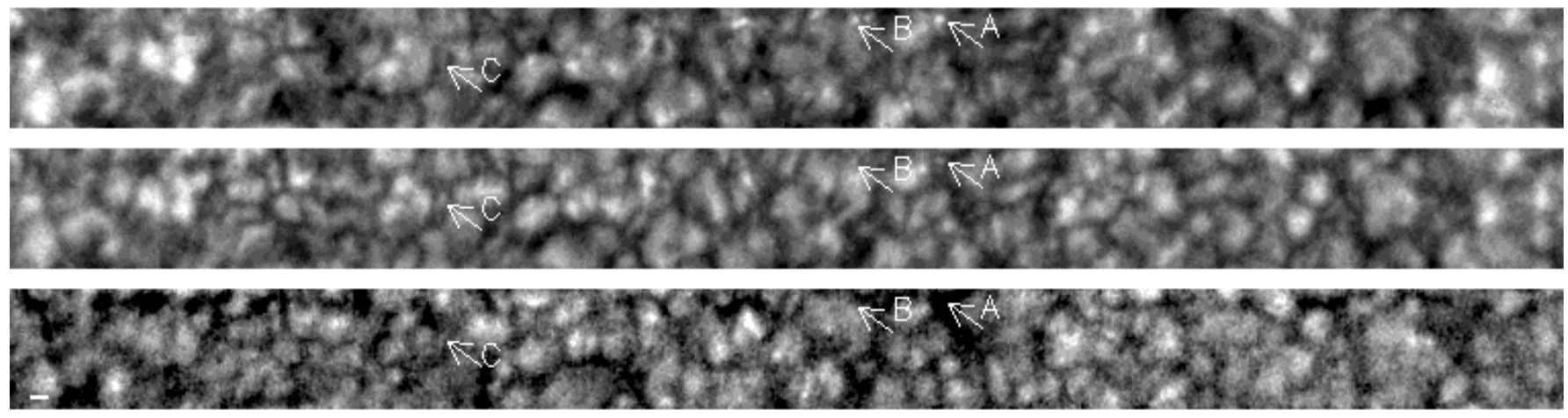

Fig. 2. Example, at $9 \mathrm{~h} 36 \mathrm{~m} 15 \mathrm{~s}$ UT, of velocity (bottom), continuum intensity (middle) and intensity at $\delta \lambda=288 \mathrm{~m} \AA$ (top), arrows indicate the location of the smallest details (A, B filigree and C granule) visible in the field fo view $\left(6^{\prime \prime} \times 76^{\prime \prime}\right)$. The small white line in the left bottom represents 1" length. The 9 bands of our observation are available at http://BASS2000. bagn. obs-mip.fr/New2001/Pages/Roudier/dpsm.html
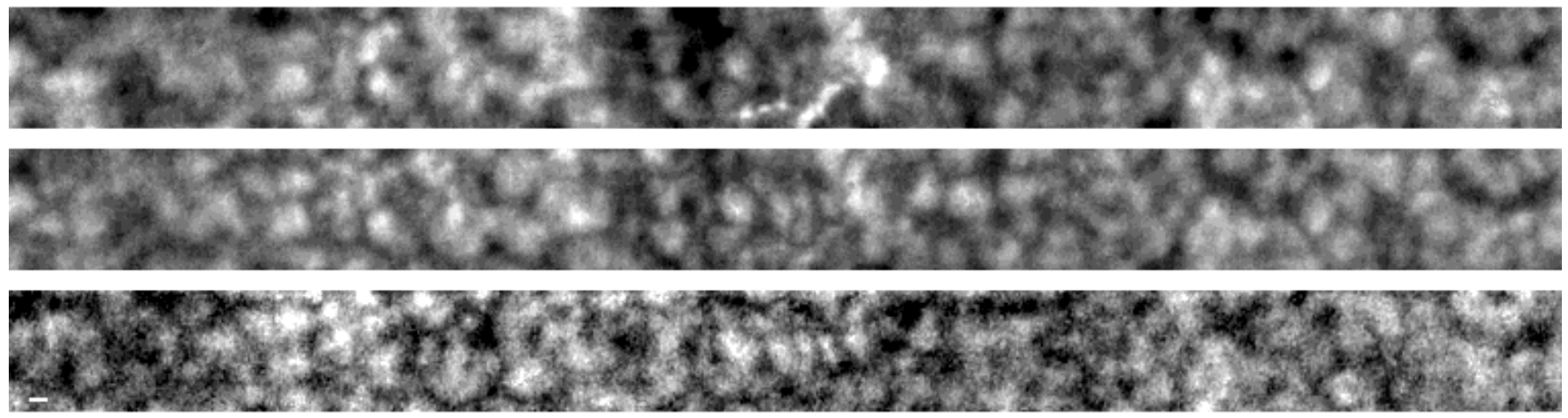

Fig. 3. Second example, at $9 \mathrm{~h} 36 \mathrm{~m} 18 \mathrm{~s}$ UT, of velocity (bottom), continuum intensity (middle) and intensity at $\delta \lambda=288 \mathrm{~m} \AA$ (top). The bright details are filigree. The small white line in the left bottom represents $1^{\prime \prime}$ length.

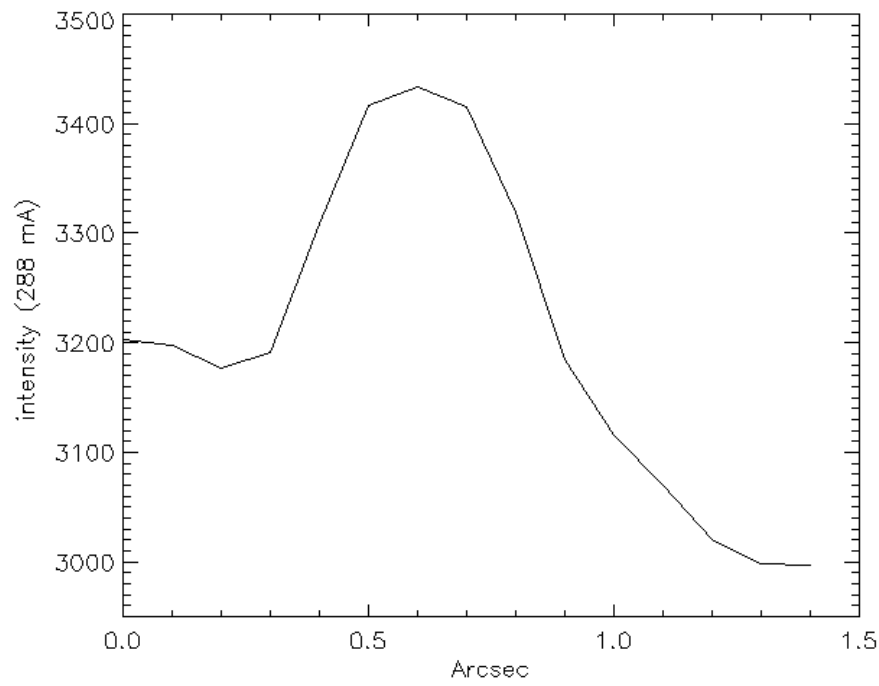

Fig. 4. Cross section of intensity at $\delta \lambda=288 \mathrm{~m} \AA$ in the central part of the small feature A (filigree) in Fig. 2.

the low frequency effects of the 5 minute oscillations ( $\mathrm{p}$ mode), we applied a simple spatial filtering. Then the granules have been identified by using the local curvature method of segmentation described in Strous (1994). This segmentation has been applied to the velocity field in order to isolate the granules in the Doppler map. Some 3750 granules were identified, with sizes in the range of $0 .{ }^{\prime \prime} 4$ to $1 . " 5$. Then for each granule,

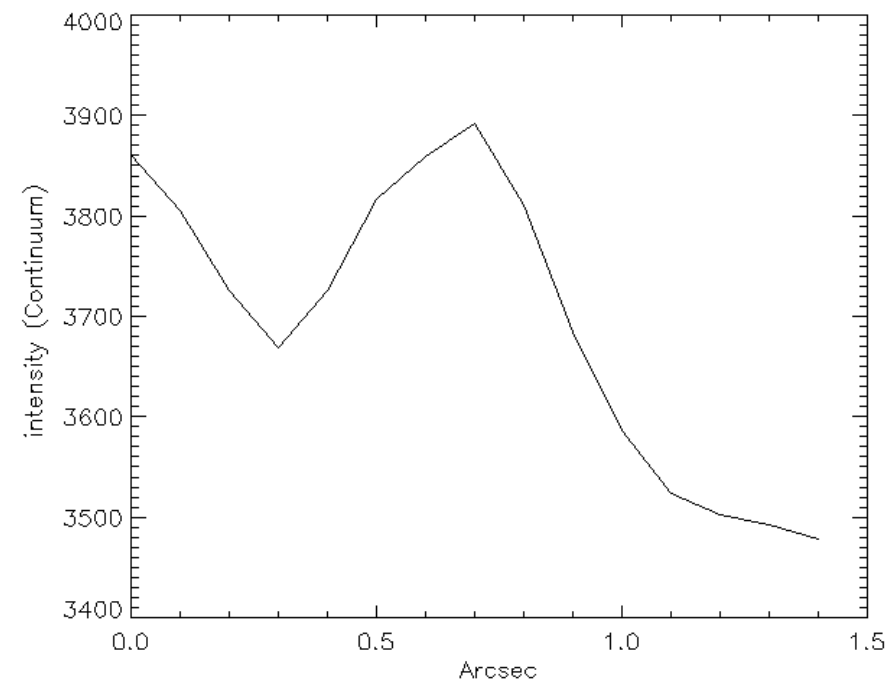

Fig. 5. Cross section of continuum intensity in the central part of the small feature A (filigree) in Fig. 2.

the mean intensity (mean velocity) was derived by averaging all the intensity (velocity) values over the granule area in the Doppler map. Finally granules smaller than 3 pixels have been removed and are considered as noise. We also took off the granules which have their center of gravity too close ( 5 pixels) to the limit of the field. 


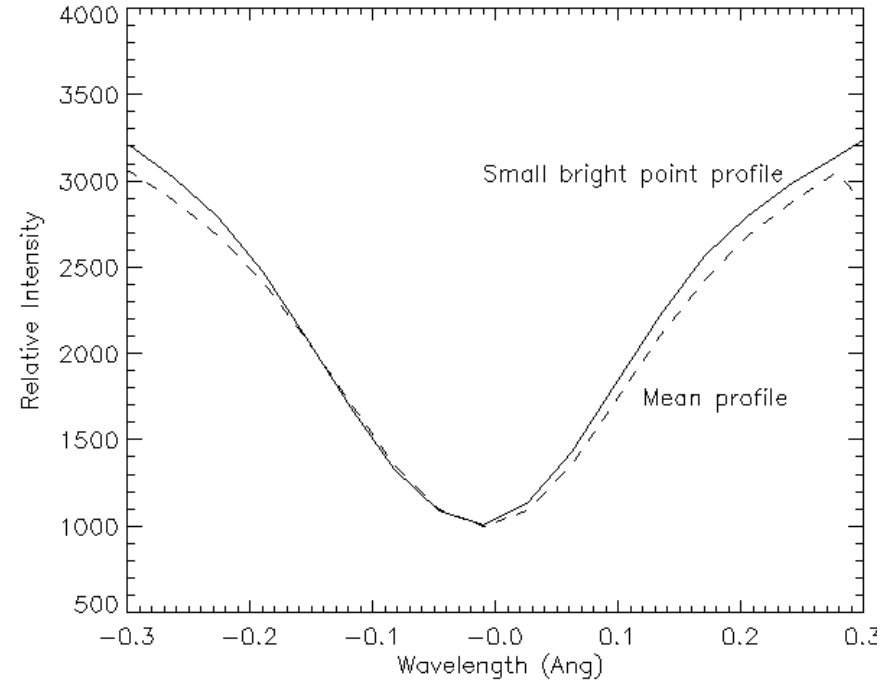

Fig. 6. Line profile in the point A of Fig. 2 and mean profile.

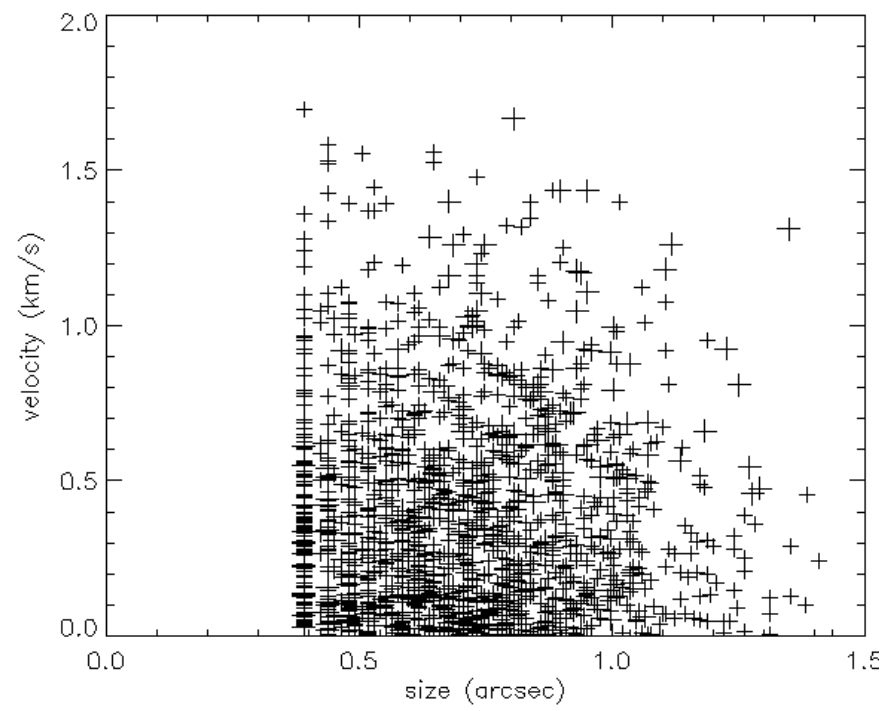

Fig. 7. Plot of the mean velocity versus to the granule size at $\delta \lambda=$ $288 \mathrm{~m} \AA$.

In Figs. 7 and 8 we plot velocity and intensity versus granule sizes down to 0.'39. This spatial resolution can be achieved thanks to the MSDP capabilities.

In our measurements, we do not observe in Fig. 7 upward velocities increasing with the granule size as previously observed by Berilli et al. (1999), and we note only that Doppler features show a large dispersion of the velocity whatever their sizes. In Fig. 8, we note a large dispersion of the brightness relatively to their size with no special trend as found by Berilli et al. (1999) and Hirzberger et al. (1997). The plot of Fig. 9 of the continuum intensity against velocity derived in the middle photosphere confirms the relation between vertical velocity and brightness. The difference between our results and those published by Berilli et al. (1999) and Hirzberger et al. (1997) may be due to our crude 5 min oscillations filtering and the presence of filigrees in the field of view.

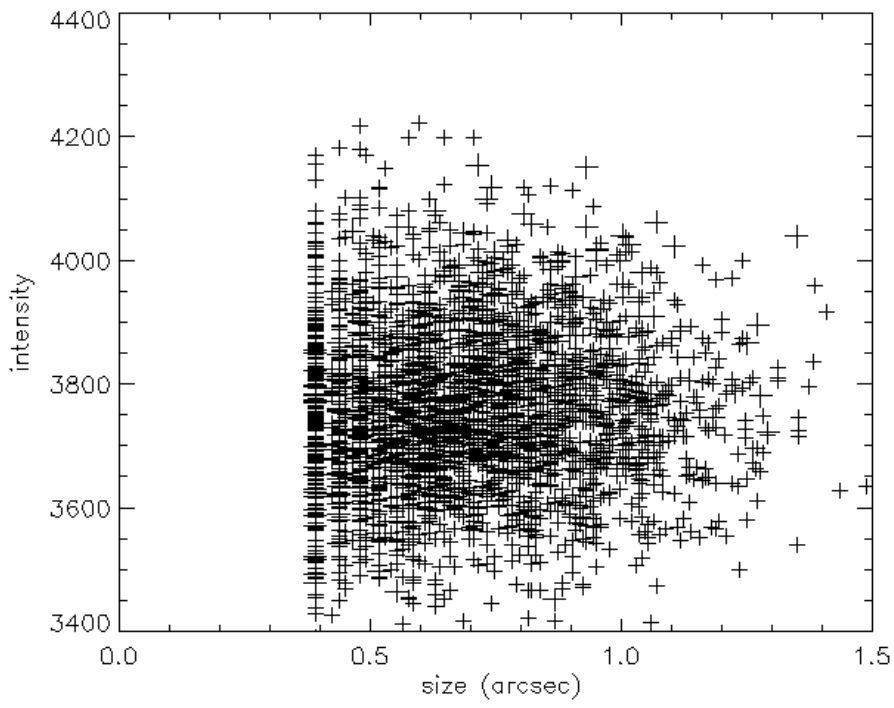

Fig. 8. Plot of the mean continuum intensity relatively to the granule size at $\delta \lambda=288 \mathrm{~m} \AA$.

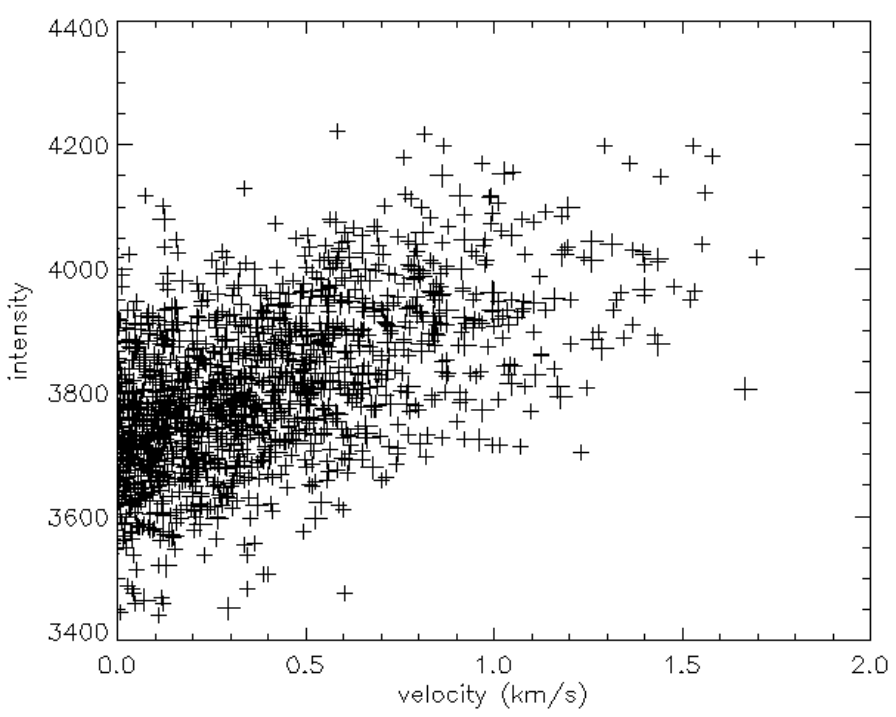

Fig. 9. Plot of the mean intensity continuum relatively to the mean velocity at $\delta \lambda=288 \mathrm{~m} \AA$.

\section{Conclusion}

The intention of this paper is to show the capabilities of the MSDP and CCD Camera system of the Pic du Midi Observatory, for 2D spectro-imagery at high spatial resolution. We are convinced that these new facilities are well suited to future detailed investigations of small scale features like flux tubes or details in the granules. New improvements, using a thinner grid in the MSDP prism box, will allow to increase the spectral resolution from $144 \mathrm{~m} \AA$ to only $50 \mathrm{~m} \AA$, allowing the observation of weak photospheric magnetic lines. The measurement of the longitudinal magnetic field will be undertaken this year with a pixel size of 0 .' 2 using a liquid cristal modulator coupled with the MSDP system. Then temporal sequences over a large field of view $\left(2^{\prime} \times 2^{\prime}\right)$ at different heights will provide information for statistical studies of the vertical structure of the granules from high to medium spatial frequencies. 
Acknowledgements. This work was supported by the Centre National de la Recherche Scientifique (CNRS, UMR 5572 and FRE 2461) and the Programme National Soleil Terre (PNST). We are grateful to N. Meunier and J. Silvester for valuable comments on the manuscript. Special thanks are due to the Pic du Midi Observatory staff for their technical assistance.

\section{References}

Berrilli, F., Florio, A., Consolini et al. 1999, A\&A, 344, L29

Bonet, J. A. 1999, Motions in Solar Atmosphere, 1-34 (The Netherlands: Kluwer Academic Publishers)

Eibe, M. T., Mein, P., Roudier, T., \& Faurobert, M. 2001, A\&A, 371, 1128
Hirzberger, J., Vazquez, M., Bonet, J. A., Hanslmeier, A., \& Sobotka, M. 1997, ApJ, 480, 406

Hirzberger, J., Koschinsky, M., Kneer, F., \& Ritter, C. 2001, A\&A, 367,1011

Hirzberger, J. 2002, A\&A, 392, 1105

Krieg, J., Kneer, F., Koschinsky, M., \& Ritter, C. 2000, A\&A, 360, 1157

Mein, P. 1981, Proc. of the Japan-France seminar on solar physics, ed. Henoux \& Moriyama, 285

Mein, P. 1991, A\&A, 248, 669

Mein, P. 2002, A\&A, 381, 271

Roudier, Th., Eibe, M. T., Malherbe et al. 2001, A\&A, 368, 652

Strous, L. H. 1994, Ph.D. Thesis (Utrecht) 